\title{
First principles phase diagram calculations for the wurtzite-structure quasibinary systems SiC-AIN, SiC-GaN and SiC-InN
}

\author{
B. P. Burton, ${ }^{1, a)}$ Steve Demers, ${ }^{2, b)}$ and A. van de Walle ${ }^{2, c)}$ \\ ${ }^{1}$ Materials Measurement Laboratory, Metallurgy Division, National Institute of Standards and Technology \\ (NIST), Gaithersburg, Maryland 20899, USA \\ ${ }^{2}$ Engineering and Applied Science Division, California Institute of Technology, 1200 E. California Blvd., MC \\ 309-81 Pasadena, California 91125, USA
}

(Received 2 November 2010; accepted 17 May 2011; published online 20 July 2011)

\begin{abstract}
The cluster-expansion method was used to perform first principles phase diagram calculations for the wurtzite-structure quasibinary systems $(\mathrm{SiC})_{1-X}(\mathrm{AlN})_{X},(\mathrm{SiC})_{1-X}(\mathrm{GaN})_{X}$ and $(\mathrm{SiC})_{1-X}(\mathrm{InN})_{X}$; and to model variations of band gaps as functions of bulk compositions and temperature. In SiC-AlN, plane wave pseudopotential formation-energy calculations predict low-energy metastable states with formation energies, $\Delta E_{\mathrm{f}} \lesssim 0.004 \mathrm{eV} / \mathrm{mole}(\mathrm{mol}=$ one cation + one anion). The crystal structures of these states are all of the form $(\mathrm{SiC})_{m}(\mathrm{AlN})_{n}(\mathrm{SiC})_{o}(\mathrm{AlN})_{p} \ldots(m, n, o, p$ integers $)$, where $(\mathrm{SiC})_{m}$ indicates $m$ SiC-diatomic-layers $\perp$ to the hexagonal $c$-axis $\left(\mathrm{c}_{\mathrm{Hex}}\right)$ and similarly for $(\mathrm{AlN})_{n},(\mathrm{SiC})_{o}$ and $(\mathrm{AlN})_{p}$. The presence of low-energy layer-structure metastable states helps to explain why one can synthesize $(\mathrm{SiC})_{1-X}(\mathrm{AlN})_{X}$ films, or single crystals with any value of $X$, in spite of the apparently strong tendency toward immiscibility. In SiC-GaN, ordered structures are predicted at $X=1 / 4,1 / 2$, and $3 / 4\left(\mathrm{Pm}, \mathrm{Pmn} 2_{1}\right.$ and $\mathrm{Pm}$, respectively). In SiC-InN, one $\mathrm{Cmc} 2_{1}$ ordered phase is predicted at $X=1 / 2$. @ 2011 American Institute of Physics. [doi:10.1063/1.3602149]
\end{abstract}

\section{INTRODUCTION}

As discussed by Gu et al. and others, ${ }^{1-4}$ the wurtzitestructure (2H, B4-Strukturbericht, space group $\left.\mathrm{P}_{3} \mathrm{mc}\right)$ $(\mathrm{SiC})_{1-X}(\mathrm{AlN})_{X}$ quasibinary ${ }^{5}$ system is particularly interesting for bandgap engineering because gaps vary from a 2.9 $\mathrm{eV}$ indirect gap in $\mathrm{SiC}$ to a $6.2 \mathrm{eV}$ direct gap in AlN (Ref. 6); and similarly for $(\mathrm{SiC})_{1-X}(\mathrm{GaN})_{X}(3.5 \mathrm{eV} \text { direct })^{7}$ and $(\mathrm{SiC})_{1-X}(\operatorname{lnN})_{X}(1.89 \mathrm{eV}$ direct $){ }^{8}$ There is a dearth of literature on the $\mathrm{SiC}-\mathrm{GaN}$ and $\mathrm{SiC}-\operatorname{lnN}$ solid solutions, but all three systems were studied to investigate the chemical systematics of ordering, and because SiC-AlN may be used as a substrate for $\mathrm{GaN}$ (Ref. 9).

The "tentative" SiC-AlN experimental phase diagram of Zangvil and Ruh ${ }^{10}$ is dominated by a wurtzite-structure solid solution above $\mathrm{T} \sim 2300 \mathrm{~K}$ and a miscibility gap below. ${ }^{10,11}$ In spite of an apparently strong tendency for immiscibility it is possible to synthesize wurtzite-structure solid solutions $(\mathrm{SiC})_{1-X}(\mathrm{AlN})_{X}$ as thin films or single crystals of arbitrary bulk composition, $X$ (Refs. 1, 2 and 9).

The first principles (FP) supercell total energy calculations and first principles phase diagram (FPPD) calculations presented here suggest a plausible explanation for the relative ease with which homogeneous solid solutions and single crystals are synthesized. Specifically, all the $(\mathrm{SiC})_{m}(\mathrm{AlN})_{n} \ldots$ supercells (Fig. 1) that are constructed of SiC- and AlN-diatomic $(001)_{w}$ layers ( $w$ for wurtzite, and similarly for all crystallographic planes or axes) have very low formation energies $\left[E_{f}<0.045 \mathrm{eV} / \mathrm{MX}-\mathrm{mol}(M=\mathrm{Si}, \mathrm{Al}, X=\mathrm{C}, \mathrm{N})\right]$ relative to $\mathrm{SiC}$ and AlN (and similarly for SiC-GaN and SiC-InN):

\footnotetext{
${ }^{a}$ Electronic mail: benjamin.burton@nist.gov. FAX: (301) 975-5334.

b)Electronic mail: steved@caltech.edu.

${ }^{c)}$ Electronic mail: avdw@alum.mit.edu.
}

$$
\Delta E_{f}=E_{S}-m E_{S i C}-n E_{A I N}
$$

Here, $E_{S}$ is the total energy of the $\left[S i_{m}, A l_{n}\right]\left(C_{m}, N_{n}\right)$ supercell; $E_{S i C}$ is the energy/mol of $\mathrm{SiC}$; and $E_{A I N}$ is the energy/ mol of AlN.

Supercell configurations, in which $\mathrm{Si}$ and $\mathrm{Al}$ mix within $(001)_{w}$-cation-layers and $C$ and $N$ mix within $(001)_{w}$-anionlayers, exhibit higher or much higher formation energies, $0.05 \mathrm{eV} / \mathrm{mol} \leq E_{f} \leq 0.65 \mathrm{eV} / \mathrm{mol}$ (Fig. 2).

Another likely source of low-energy metastable states, especially on the $\mathrm{SiC}$-rich side of the diagram, is the presence of stacking faults perpendicular to the hexagonal $c$-axis $\left(\mathrm{c}_{H e x}\right.$;

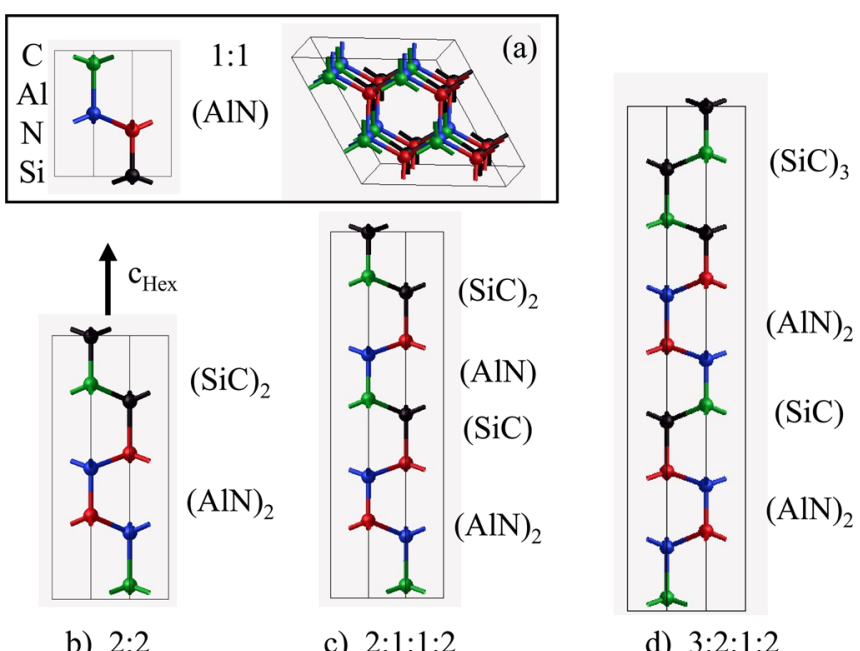

FIG. 1. (Color online) Representations of some $(001)_{w}$ layer structures with $X=1 / 2$ : (a) the simplest $(\mathrm{SiC})_{1}(\mathrm{AlN})_{1} 1: 1$-supercell which is predicted to be the lowest energy configuration at $X=1 / 2$ [viewed with $(001)_{w}$ almost in the plane of the page. (b) $(\mathrm{SiC})_{2}(\mathrm{AlN})_{2} \quad 2: 2$-supercell; (c) $(\mathrm{SiC})_{2}(\mathrm{AlN})_{2}$ $(\mathrm{SiC})_{1}(\mathrm{AlN})_{1}$ 2:1:1:2-supercell; and $(\mathrm{d})(\mathrm{SiC})_{3}(\mathrm{AlN})_{2}(\mathrm{SiC})_{1}(\mathrm{AlN})_{2}$ 3:2:1:2supercell $($ online, black $=\mathrm{Si}$; blue $=\mathrm{Al}$; green $=\mathrm{C} ;$ red $=\mathrm{N}$ ). 

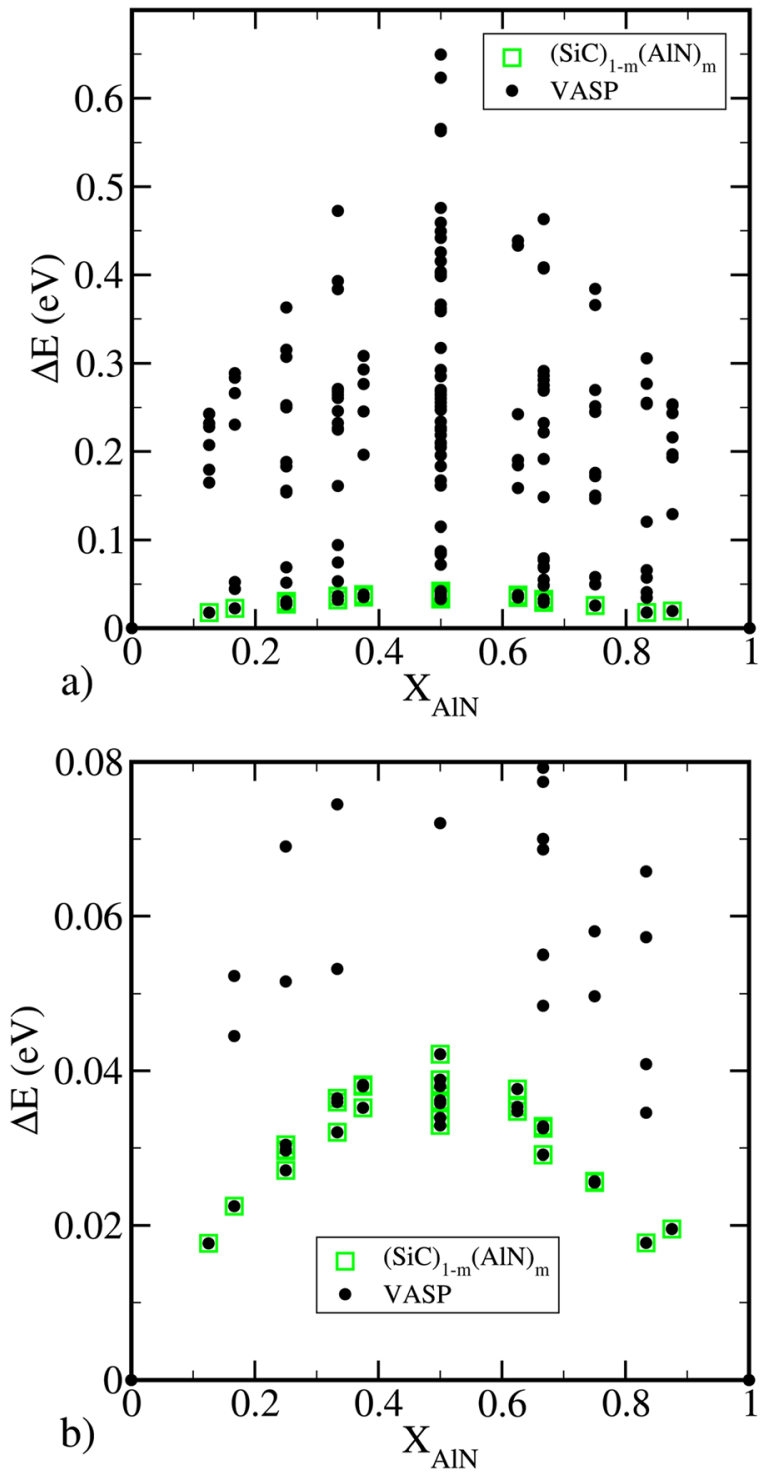

FIG. 2. (Color online) Calculated, VASP, formation energies, $\Delta E_{f}$, for the SiC-AlN quasibinary (solid circles, black online). The $\Delta E_{f}$ for $(\mathrm{SiC})_{m}$ $(\mathrm{AlN})_{n} \ldots$ layer structures (layers $\perp c_{\mathrm{Hex}}$ ) are indicated by open squares (green online): (a) The full set of $177 \Delta E_{f}$; (b) the low energy part of the distribution. Note the unusual distribution of formation energies: low- $\Delta E_{f}$ metastable states. Note the unusual distribution of formation energies: low- $\Delta E_{f}$ metastable states; a relatively depopulated gap; and a very wide range of high-energy configurations, $0.10 \mathrm{eV} / \mathrm{mol} \leq E_{f} \leq 0.65 \mathrm{eV} / \mathrm{mol}$.

$\langle 001\rangle_{\omega}$ ) that create sphalerite-type $\mathrm{ABC} \ldots$ stacking rather than wurtzite type ABAB. stacking (sphalerite= zincblend, 3C, B3-Strukturbericht, space group F43m). ${ }^{12}$ However, a treatment that compares sphalerite- and wurtzite-structure solutions is beyond the scope of this study.

Results for the S-C-GaN and SiC-InN systems evince no obvious chemically-systematic trends. Three ground-state phases are predicted in SiC-GaN (at $X=1 / 4,1 / 2$ and 3/4), and only one in $\mathrm{SiC}-\mathrm{InN}$ (at $X=1 / 2$ ).

\section{COMPUTATIONAL METHODOLOGY}

\section{A. Formation energy calculations}

Formation energies, $\Delta E_{f}$ (Fig. 2) were calculated for fully relaxed wurtzite-structure $\mathrm{SiC}, \mathrm{AlN}$ and 175 ordered
$\left[S i_{m}, A l_{n}\right]\left(C_{m}, N_{n}\right)$ supercells; where square brackets and parentheses, respectively, indicate that $\mathrm{Si}$ and $\mathrm{Al}(\mathrm{Ga}, \mathrm{In})$ mix on one set of [cation] sites, while $C$ and $N$ mix on a mutually exclusive set of (anion) sites; and similarly for GaN plus 117 $\left[S i_{m}, G a_{n}\right]\left(C_{m}, N_{n}\right)$ supercells; and $\mathrm{InN}$ plus $85\left[S i_{m}, I n_{n}\right]$ $\left(C_{m}, N_{n}\right)$ supercells.

All total energy calculations were performed with the Vienna ab initio simulation program [VASP, version 445 (Refs. 13-15)] using all electron projector augmented wave (PAW) pseudopotentials, with the generalized gradient approximation (GGA) for exchange and correlation energies; specifically, the $\mathrm{Si}, \mathrm{Al}, \mathrm{Ga}_{d}, \mathrm{In}_{d}, \mathrm{C}_{s}$ and $\mathrm{N}_{h}$ PAW potentials. Electronic degrees of freedom were optimized with a conjugate gradient algorithm. Both cell constants and ionic positions were fully relaxed for each structure.

Total energy calculations were converged with respect to $k$-point meshes by using the equivalent of 664 , or greater, for a four-atom (two mol) wurtzite-structure cell. A $900 \mathrm{eV}$ energy cutoff was used, in the "high precision" option which guarantees that absolute energies are converged to within a few meV/mol: a few tenths of a $\mathrm{kJ} / \mathrm{mol}$ of exchangeable atoms [Si exchangeable with $\mathrm{Al}]$ and $(C$ with $N)$; one $\mathrm{mol}=\mathrm{MX}(\mathrm{M}=\mathrm{Si}, \mathrm{Al}$, Ga or In; $\mathrm{X}=\mathrm{C}, \mathrm{N})$. Residual forces were typically of the order of $0.02 \mathrm{eV}$ or less.

\section{B. SiC-AIN}

Calculated formation energies, $\Delta E_{f}$, for $\mathrm{SiC}, \mathrm{AlN}$ and $175\left[S i_{m}, A l_{n}\right]\left(C_{m}, N_{n}\right)$ supercells are plotted in Fig. 2 $(\bullet)$, where values for $\Delta E_{f}$ are normalized per MX-mol of exchangeable ions.

\section{The cluster expansion hamiltonian}

The cluster expansion (CE), ${ }^{16}$ is a compact representation of the configurational total energy. In a two-component A-B alloy, the solid solution configuration is described by pseudospin occupation variables $\sigma_{i}$, which take values $\sigma_{i}=-1$ when site- $i$ is occupied by atom-A and $\sigma_{i}=+1$ when site- $i$ is occupied by atom-B. Extensions to higherorder systems such as the four component $(\mathrm{SiC})_{1-X}(\mathrm{AlN})_{X}$ system, are described by Sanchez et $a l . ;{ }^{16}$ as well as some examples of applications described in Refs. 17-20.

The CE parameterizes the configurational energy, per exchangeable cation, as a polynomial in pseudospin occupation variables:

$$
E(\sigma)=\sum_{\ell} m_{\ell} J_{\ell}\left\langle\prod_{i \in \ell^{\prime}} \sigma_{i}\right\rangle
$$

Cluster $\ell$ is defined as a set of lattice sites. The sum is taken overall clusters $\ell$ that are not symmetrically equivalent in the high- $T$ structure space group, and the average is taken overall clusters $\ell^{\prime}$ that are symmetrically equivalent to $\ell$. Coefficients $J_{\ell}$ are called effective cluster interactions, ECI, and the multiplicity of a cluster, $m_{\ell}$, is the number of symmetrically equivalent clusters, divided by the number of cation sites. The ECI are obtained by fitting a set of VASP FP 
calculated structure energies, $\left\{E_{S t r}\right\}$. The resulting CE can be improved as necessary by increasing the number of clusters $\ell$ and/or the number of $E_{S t r}$ used in the fit.

Fitting was performed with the Alloy Theoretic Automated Toolkit (ATAT) ${ }^{15,21-23}$ which automates most of the tasks associated with the construction of a CE Hamiltonian. A complete description of the algorithms underlying the code can be found in Ref. 22. The most important steps are: (1) Selecting which first principles (FP) structure energies to calculate, which is done in a way that minimizes the statistical variance of the estimated ECI; (2) Automatically selecting which clusters to include in the expansion by minimizing the cross-validation score, $\mathrm{CV}$ :

$$
(C V)^{2}=\frac{1}{N} \sum_{S t r=1}^{N}\left(E_{S t r}-\hat{E}_{S t r}\right)^{2}
$$

where $E_{1}, \ldots E_{N}$ denote the structural energies calculated from FP and $\hat{E}_{S t r}$ is the energy of structure Str predicted from a $\mathrm{CE}$ that was fit to the remaining $N-1$ energies. This criterion ensures that the chosen set of clusters maximizes the predictive power of the $\mathrm{CE}$ for any structure, whether or not it is included in the fit. This is an improvement relative to the standard mean square error criterion which only minimizes the error for structures that are included in the fit.

Although as noted in point (1) above, the maps code automatically selects structures for which the $E_{S t r}$ are calculated, but it also has a bias in favor of smaller supercells to minimize computation time. Thus, the largest supercells it generated had eight atoms. The lowest energy states among maps-generated cells were all $(\mathrm{SiC})_{m}(\mathrm{AlN})_{n} \ldots$ layer structures in which the SiC- and AlN-double-layers are $\perp c_{H e x}$. Formation energies for these structures were typically $\Delta E_{f}<0.04 \mathrm{eV} / \mathrm{mol}(7 \mathrm{~kJ} /$ mol) [Figs. 2(a) and 2(b)]. To test the generality of this observation, 27 additional $(\mathrm{SiC})_{m}(\mathrm{AlN})_{n} \ldots$-calculations were performed. These are structures in which $\mathrm{Si}-\mathrm{C}$ and $\mathrm{Al}-\mathrm{N}$ bonds are maximized, and $\Delta E_{f}<0.04 \mathrm{eV} / \mathrm{mol}$ holds for the whole set [(open squares, green online, Fig. 2(b)]. For this set, $\left\{\Delta E_{f}\right\}$ describe an approximate parabola which strongly suggests that no intermediate ordered phases are stable. Also, $11(\mathrm{AlC})_{\max }$ $(\mathrm{SiN})_{\max }$ structure energies were calculated; i.e., 12-atom/cell layer structures in which $\mathrm{Al}-\mathrm{C}$ and $\mathrm{Si}-\mathrm{N}$ nearest neighbor bonds are maximized. For this subset, $0.15 \mathrm{eV} / \mathrm{mol}<\Delta E_{f}$ $<0.6 \mathrm{eV} / \mathrm{mol}\left(30 \mathrm{~kJ} / \mathrm{mol}<\Delta E_{f}<125 \mathrm{~kJ} / \mathrm{mol}\right)$.

\section{Cluster expansion of band gaps}

To model band-gaps in disordered solid solutions, bandgap-cluster-expansions, $\mathrm{CE}_{G a p}$, were fit to hybrid functional $^{24}$ band-gap calculations for all the same structures (virtual compounds) that were used to calculate phase diagrams. Typically, GGA greatly underpredicts semiconductor band gaps. Therefore, band gaps were calculated with hybrid density functionals, in which a specific amount of exact, non-local hartree fock (HF) exchange energy is mixed into the semi-local GGA portion. ${ }^{24}$ This reduces the "self-interaction error" by more realistically modeling electron localization, and yields a more accurate bandgap. The Heyd et al. $(\mathrm{HSE})^{24}$ functional, was used because it is highly efficient, owing to its separation of the exact HF-exchange into shortand long-range parts (via a 'screening length' parameter); where the slowly decaying long-range exchange interactions are replaced with their GGA counterparts. ${ }^{24}$ Specifically, the HSE06 functional, which uses $25 \%$ HF-exchange mixing and a screening length of $0.2 \AA^{-1},{ }^{25}$ was used because this approximation yields good agreement with experiment for a wide range of semiconductors. ${ }^{25}$

The HSE06 functional was used to calculate band gaps for all the structures that were used to fit the CE Hamiltonians for phase diagram calculations; the $C E_{G a p}$ were then used to calculate band gaps for disordered solid solutions as functions of bulk composition and temperature.

In the systems studied here, the electronic structures of virtual compounds exhibit wide variability (including many near-zero bandgaps), which makes it difficult to fit a $C E_{G a p}$ that works well for all configurational states. Most of this variability, however, is concentrated in the high-energy structures which are rarely sampled at thermal equilibrium. Therefore, high-energy structures were excluded (i.e., $\Delta E_{s t r}$ $>0.11 \mathrm{eV}$, for SiC-AlN and SiC-GaN; and $\Delta E_{\text {str }}>0.14 \mathrm{eV}$ for $\mathrm{SiC}-\mathrm{InN}$ ). In addition, a few of the remaining structures with band gaps less than $0.1 \mathrm{eV}$ were also excluded. For $\mathrm{SiC}-\mathrm{InN}, \mathrm{SiC}$-rich structures are also excluded, because the system exhibits a wide miscibility gap in that region.

To ensure that end member band gaps (i.e., gaps for $\mathrm{SiC}, \mathrm{AlN}, \mathrm{GaN}$ and $\mathrm{InN}$ ) are accurately reproduced, they are assigned increased weight in the fit (five instead of one). The $C E_{\text {Gap }}$ are optimized with a $\mathrm{CV}$ criterion and contain six pairs (for SiC-AlN), 13 pairs (for SiC-GaN) and seven pairs (for $\mathrm{SiC}-\mathrm{InN}$ ). Multibody terms did not yield improved predictive power. The resulting optimal $C E_{G a p}$ expansions exhibit CV-scores of $0.21 \mathrm{eV}$ (for SiC-AlN), $0.32 \mathrm{eV}$ (for SiC$\mathrm{GaN}$ ) and $0.37 \mathrm{eV}$ (for $\mathrm{SiC}-\mathrm{InN}$ ). These $C E_{\text {Gap }}$ are used, with the correlations obtained from Monte Carlo simulations, to predict band gaps of solid solutions that fully account for the effects of potential short-range order. Figures 4(a)-4(c) show the predicted band gaps for the solid solutions as functions of temperature, plus the band gaps of some perfectly ordered phases (circles) and random solid solutions $(T=\infty)$, for comparison. Note, that the inclusion of short-range-order effects (i.e., $\mathrm{T}<\infty$ ) has little effect in the system SiC-AlN, but dramatically changes results in SiC-GaN and SiC-InN. Also, in SiC-GaN, the bandgap of the solid solution approaches the gaps of ordered GS phases when short-rangeorder effects are included.

\section{RESULTS}

\section{A. Ground-state analyses}

\section{1. $S i C-A I N$}

In Figs. 2 and 3(a) and 3(b), all $\Delta E_{f}>0$, indicates a miscibility gap, but the range of $\Delta E_{f}$ values, $0.02 \mathrm{eV} /$ $\mathrm{mol} \lesssim \Delta E_{f} \lesssim 0.6 \mathrm{eV} / \mathrm{mol}\left(3 \mathrm{~kJ} / \mathrm{mol} \lesssim \Delta E_{f} \lesssim 125 \mathrm{~kJ} / \mathrm{mol}\right)$ is unusually large; e.g., the comparable range in wurtzite based miscibility-gap system, GaN-InN, is $0.03 \mathrm{eV} / \mathrm{mol}$ $\lesssim \Delta E_{f} \lesssim 0.18 \mathrm{eV} / \mathrm{mol}$ and the calculated consolute temperature is $\mathrm{T}_{C} \approx 1840 \mathrm{~K}$. 

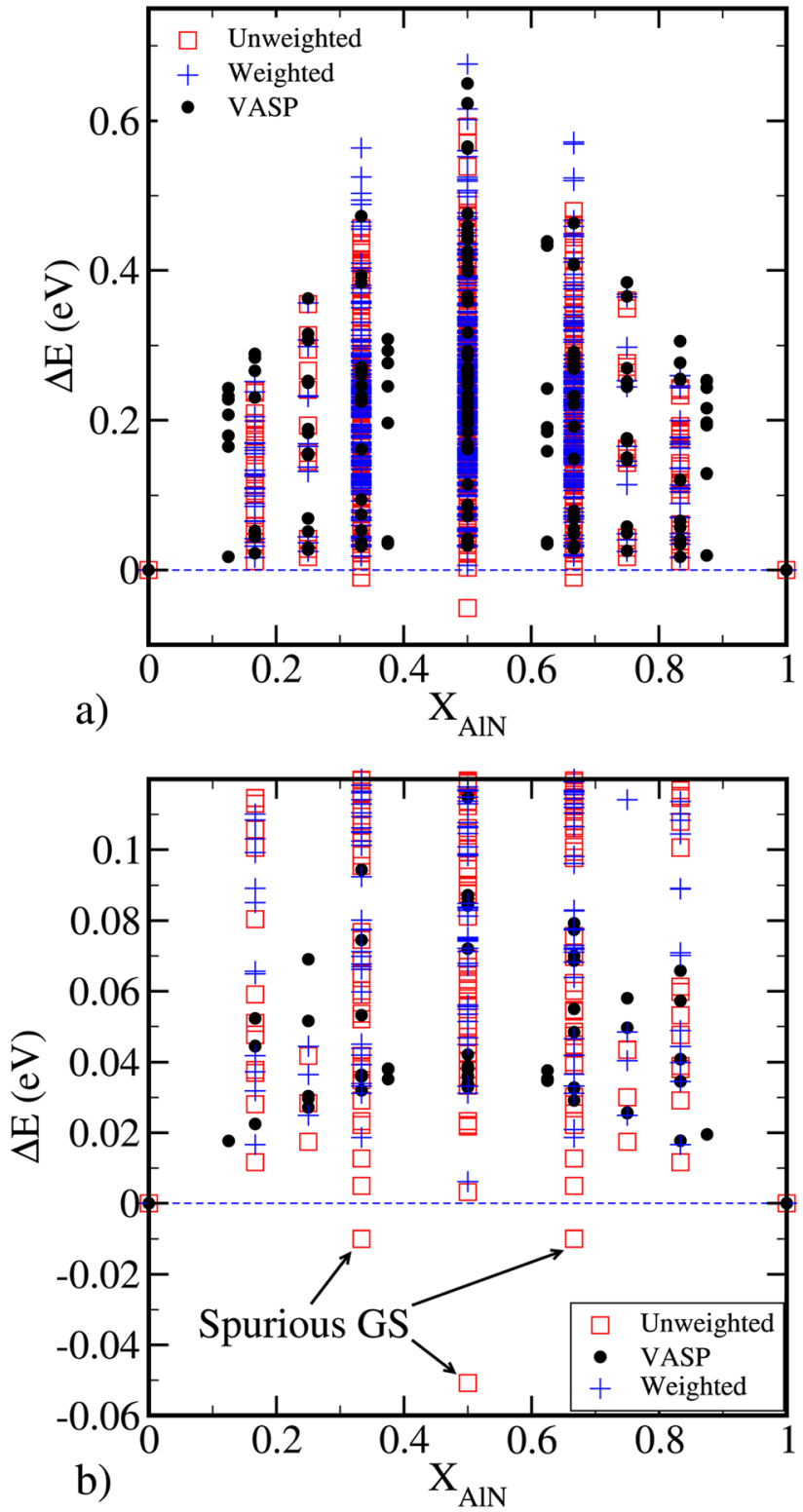

FIG. 3. (Color online) Cluster expansion based GS analyses for unweighted(open square, red online), and weighted- (plus signs, blue online) fits; VASP $\Delta E_{f}$ values are indicated by solid circles (black online).

Ground-state (GS) analyses were performed for all configurations with 16 atoms or fewer. Each predicted GS phase was tested by a VASP calculation, and all yielded $\Delta E_{f}>0$, hence, the very large number of $\Delta E_{f}$ that were calculated. Because the $\Delta E_{f}$ distribution [Figs. 2(a) and 2(b)] spreads over such a broad range of energies, unweighted CE fits always included spurious GS; e.g., the three open squares with $\Delta E_{f}<0$ in Figs. 3(a) and 3(b) for which VASP calculations yielded $\Delta E_{f}>0$. Hence, to obtain a fit without spurious GS, a weighted CE-fit was used, one which gave greater weight to the low-energy layer-structures (plus signs, and shown in blue online) in Figs. 3(a) and 3(b). Although the weighted fit avoids spurious GS, the lowest energy configuration at $X=0.5$ is very close to $\Delta E_{f}=0$, and this suggests that the weighted CE-fit is not entirely satisfactory; hence, a cluster expansion that was only fitted to $\{\Delta E \leq\} 0.1 \mathrm{eV}$

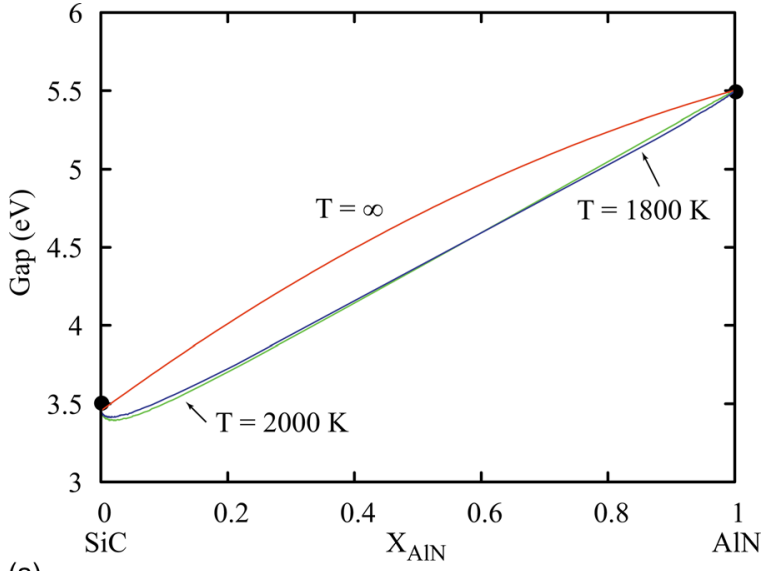

(a)

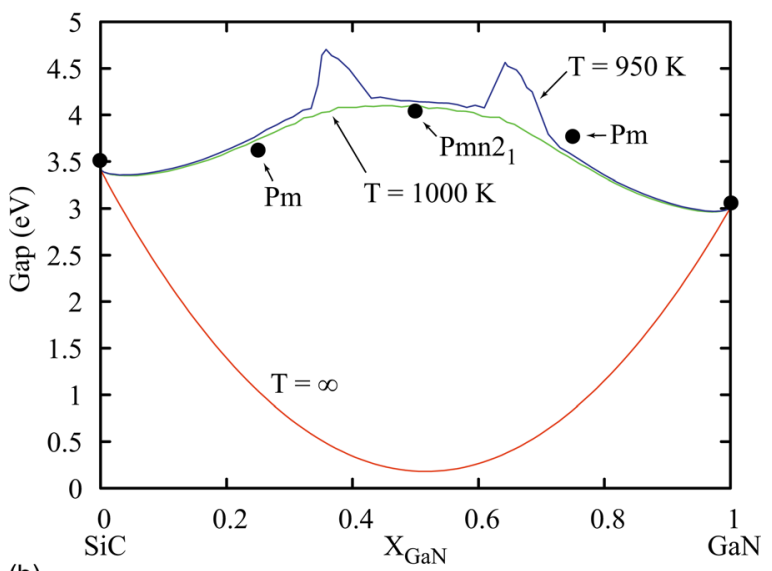

(b)

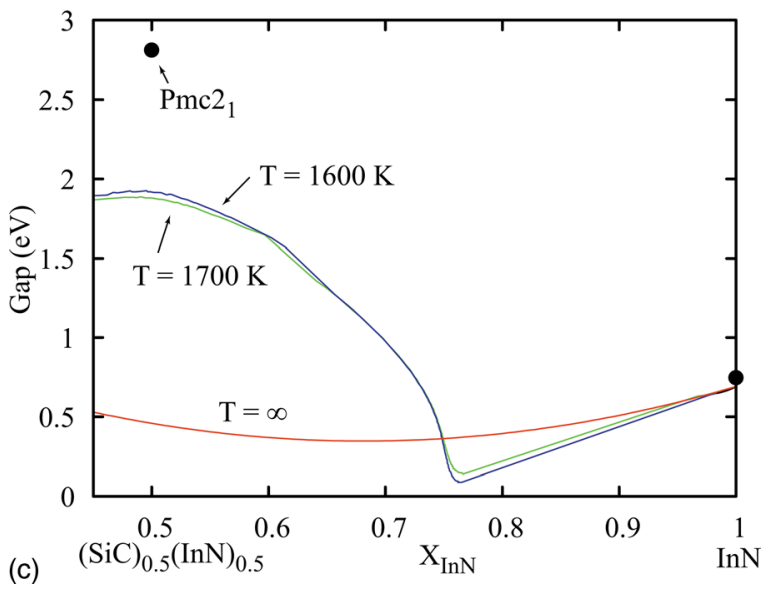

FIG. 4. (Color online) Cluster expansions of band gaps based on hybridfunctional bandgap calculations: (a) SiC-AlN; (b) SiC-GaN; (c) (SiC) $0_{0 .}^{-}$ $(\mathrm{InN})_{0.5}$-InN, only the InN-rich half of this system was modeled because the broad miscibility gap on the SiC-rich side extends to such high-T.

ensure that the CE faithfully reproduces the energies of the states most frequently visited at equilibrium.

\section{SiC-GaN}

Ground state analysis for SiC-GaN, Fig. 5(a) and 5(b), predicts three ordered GS-phases at $X=1 / 4,1 / 2$ and 3/4. Their fully relaxed crystal structures are described in Table I, and their space groups were determined with the FINDSYM program [15]. The complimentary $\mathrm{Si}_{3} \mathrm{GaC}_{3} \mathrm{~N}$ - and $\mathrm{SiGa}_{3}$ $\mathrm{CN}_{3}$-GS-structures at $X=1 / 4$ and $3 / 4$, respectively, have the 

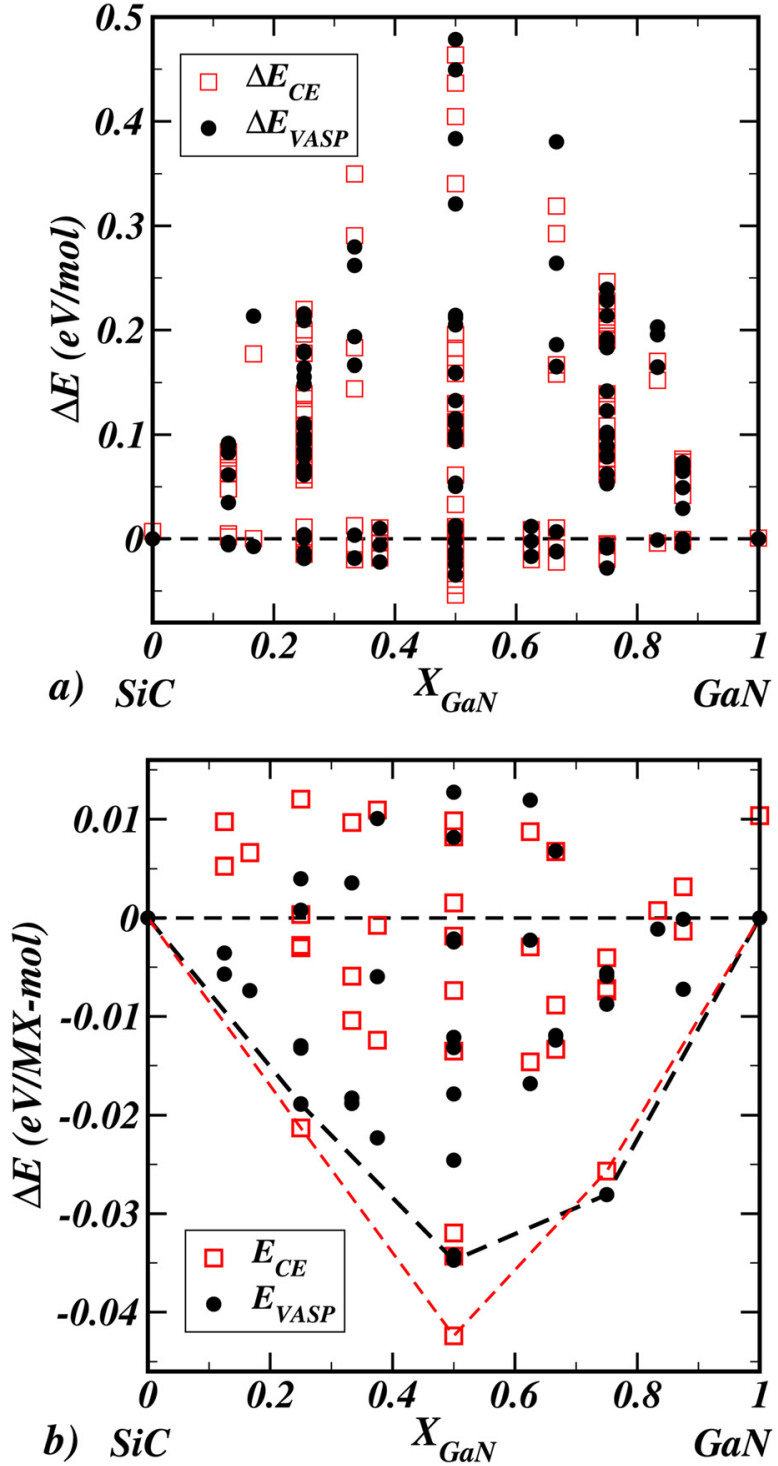

FIG. 5. (Color online) VASP and corresponding CE values for $\Delta E_{f}$ in SiCGaN (color online: $\Delta E_{V A S P}=$ solid black circles; $\Delta E_{C E}=$ open red squares; dashed lines indicate convex hulls): (a) the energy distribution in SiC-GaN is similar to that in $\mathrm{SiC}-\mathrm{AlN}$, i.e., a wide $\Delta E_{f}$-range; but unlike $\mathrm{SiC}-\mathrm{AlN}$, (b) the SiC-GaN distribution includes many $\Delta E_{f}<0$, which indicates the presence of ordered phases.

same Pm monoclinic structures: in $\mathrm{Si}_{3} \mathrm{GaC}_{3} \mathrm{~N}, \mathrm{SiC}-(001)_{w^{-}}$ double-layers alternate with $\mathrm{Si}_{1 / 2} \mathrm{Ga}_{1 / 2} \mathrm{C}_{1 / 2} \mathrm{~N}_{1 / 2}$-mixed-double-layers; similarly, in $\mathrm{SiGa}_{3} \mathrm{CN}_{3}$, GaN-double-layers alternate with $\mathrm{Si}_{1 / 2} \mathrm{Ga}_{1 / 2} \mathrm{C}_{1 / 2} \mathrm{~N}_{1 / 2}$-mixed-double-layers $\perp$ $\langle 001\rangle_{\omega}$. The predicted GS at $X=1 / 2$ is orthorhombic, Pmn $2_{1}$, with all $(001)_{w}$ basal layers mixed, and alternating SiC- and GaN-double-layers in $(210)_{w}$.

\section{3. $\operatorname{SiC}-\operatorname{In} N$}

Only one ordered GS-phase is predicted in $\mathrm{SiC}$-InN. Its crystal structure is drawn in Fig. 6(c), and described in Table I. The $\Delta E_{V A S P}$ and corresponding $\Delta E_{C E}$ values are plotted in Figs. 7(a) and 7(b). Their distribution is similar to that of $\mathrm{SiC}-\mathrm{GaN}$, but more compact with $0.05 \mathrm{eV} / \mathrm{mol} \leq E_{f} \leq 0.40$ $\mathrm{eV} / \mathrm{mol}$, except for one outlier at $\Delta E_{f} \approx 0.5 \mathrm{eV}$, and only three structures with $\Delta E_{f}<0$.
TABLE I. Crystal structure parameters for the observed (wurtzite) and (fully relaxed) predicted phases in the SiC-AlN, SiC-GaN and SiC-InN quasibinary systems. The unit cells of predicted $X=1 / 4, X=1 / 2$ and $X=3 / 4$ phases are all $0, \overline{1}, 0 ; 0,0, \overline{1} ; 2,1,0$ supercells of wurtzite. Cell constants are given in $\AA$ and degrees; and all experimental values are from Pearson's Handbook. ${ }^{26}$

\begin{tabular}{|c|c|c|c|c|}
\hline System & $X$ & $\begin{array}{c}\text { SG } \\
\text { IT number } \\
\text { Pearson Symbol }\end{array}$ & $\begin{array}{c}\text { Experimental } \\
\text { cell constants } \\
\AA\end{array}$ & $\begin{array}{c}\text { Calculated } \\
\text { cell constants } \\
\AA\end{array}$ \\
\hline & & $\mathrm{P}_{3} \mathrm{mc}$ & $\mathrm{a}=3.0763$ & $\mathrm{a}=3.0919$ \\
\hline \multirow[t]{2}{*}{$\mathrm{SiC}$} & 0 & $\begin{array}{l}186 \\
\mathrm{hP} 4\end{array}$ & $\mathrm{c}=5.0480$ & $\mathrm{c}=5.0745$ \\
\hline & & $\mathrm{P}_{3} \mathrm{mc}$ & $\mathrm{a}=3.110$ & $\mathrm{a}=3.121$ \\
\hline AlN & 1 & $\begin{array}{l}186 \\
\text { hP4 }\end{array}$ & $c=4.980$ & $c=4.998$ \\
\hline $\mathrm{GaN}$ & 1 & $\begin{array}{c}\mathrm{P}_{3} \mathrm{mc} \\
186 \\
\mathrm{hP} 4\end{array}$ & $\begin{array}{l}a=3.190 \\
c=5.189\end{array}$ & $\begin{array}{l}a=3.211 \\
c=5.233\end{array}$ \\
\hline $\mathrm{InN}$ & 1 & $\begin{array}{c}\mathrm{P} 6_{3} \mathrm{mc} \\
186 \\
\mathrm{hP} 4\end{array}$ & $\begin{array}{l}a=3.540 \\
c=5.704\end{array}$ & $\begin{array}{l}\mathrm{a}=3.211 \\
\mathrm{c}=5.726\end{array}$ \\
\hline $\mathrm{SiGaCN}$ & $1 / 2$ & $\begin{array}{c}\mathrm{Pmn} 2_{1} \\
31 \\
\text { oP8 }\end{array}$ & & \\
\hline cell constants: & atom & Wyckoff site & $y$ & z \\
\hline $\mathrm{a}=3.137 \alpha=90$ & $\mathrm{Si}$ & $2 \mathrm{a}$ & 0.4177 & -0.4967 \\
\hline $\mathrm{b}=5.441 \beta=90$ & $\mathrm{Ga}$ & $2 a$ & 0.0905 & -0.0077 \\
\hline \multirow[t]{3}{*}{$c=5.152 \gamma=90$} & $\mathrm{~N}$ & $2 \mathrm{a}$ & 0.4341 & 0.1364 \\
\hline & $\mathrm{C}$ & $2 \mathrm{a}$ & 0.1017 & -0.3961 \\
\hline & & $\mathrm{Cmc} 2_{1}$ & & \\
\hline SiInCN & $1 / 2$ & $\begin{array}{c}26 \\
\text { oP8 }\end{array}$ & & \\
\hline cell constants: & atom & Wyckoff site & $y$ & $z$ \\
\hline $\mathrm{a}=3.236 \alpha=90$ & $\mathrm{Si}$ & $2 \mathrm{a}$ & 0.1528 & 0.4968 \\
\hline $\mathrm{b}=5.863 \beta=90$ & In & $2 \mathrm{a}$ & 0.3318 & -0.0023 \\
\hline \multirow[t]{3}{*}{$c=5.332 \gamma=90$} & $\mathrm{~N}$ & $2 \mathrm{a}$ & 0.2971 & -0.4162 \\
\hline & $\mathrm{C}$ & $2 \mathrm{a}$ & 0.1168 & 0.1578 \\
\hline & & $\mathrm{Pm}$ & & \\
\hline \multirow[t]{2}{*}{$\mathrm{Si}_{3} \mathrm{GaC}_{3} \mathrm{~N}$} & $1 / 4$ & 6 & & \\
\hline & & mP8 & & \\
\hline cell constants: & atom & Wyckoff site & $x$ & $z$ \\
\hline $\mathrm{a}=5.105$ & $\mathrm{Si}$ & $1 \mathrm{a}$ & -0.0081 & 0.3373 \\
\hline $\mathrm{b}=3.111$ & $\mathrm{Si}$ & $1 \mathrm{a}$ & -0.4973 & -0.3348 \\
\hline $\mathrm{c}=5.400$ & $\mathrm{Si}$ & $1 b$ & -0.0015 & -0.1692 \\
\hline$\alpha=90$ & $\mathrm{Ga}$ & $1 b$ & 0.4915 & 0.1633 \\
\hline$\beta=89.747$ & $\mathrm{C}$ & $1 b$ & 0.0957 & 0.1692 \\
\hline \multirow[t]{4}{*}{$\gamma=90$} & $\mathrm{C}$ & $1 b$ & -0.3709 & -0.1847 \\
\hline & $\mathrm{C}$ & $1 \mathrm{a}$ & 0.1283 & -0.3356 \\
\hline & $\mathrm{N}$ & $1 \mathrm{a}$ & -0.3658 & 0.3545 \\
\hline & & $\mathrm{Pm}$ & & \\
\hline \multirow[t]{2}{*}{$\mathrm{SiGa}_{3} \mathrm{CN}_{3}$} & $3 / 4$ & 6 & & \\
\hline & & $\mathrm{mP8}$ & & \\
\hline cell constants: & atom & Wyckoff site & $x$ & z \\
\hline $\mathrm{a}=5.184$ & $\mathrm{Si}$ & $1 \mathrm{a}$ & -0.0024 & -0.1650 \\
\hline $\mathrm{b}=3.168$ & $\mathrm{Ga}$ & 1a & -0.0054 & 0.3363 \\
\hline$c=5.502$ & $\mathrm{Ga}$ & $1 b$ & 0.4953 & 0.1619 \\
\hline$\alpha=90$ & $\mathrm{Ga}$ & $1 \mathrm{a}$ & 0.4979 & -0.3305 \\
\hline$\beta=90.326$ & $\mathrm{C}$ & 1a & 0.1112 & -0.3139 \\
\hline \multirow[t]{3}{*}{$\gamma=90$} & $\mathrm{~N}$ & $1 \mathrm{a}$ & -0.3860 & 0.3310 \\
\hline & $N$ & $1 b$ & 0.1109 & 0.1445 \\
\hline & $N$ & $1 \mathrm{~b}$ & -0.3494 & -0.1643 \\
\hline
\end{tabular}




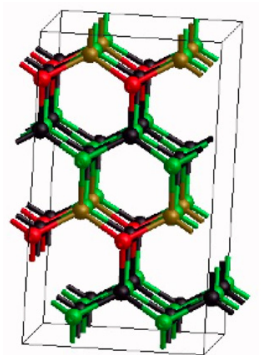

(a)

$\mathrm{Si}_{3} \mathrm{GaC}_{3} \mathrm{~N}$ and

$\mathrm{SiGa}_{3} \mathrm{CN}_{3}$

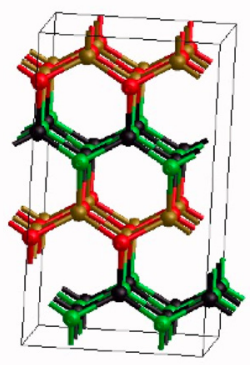

(b)

$\mathrm{SiGaCN}$

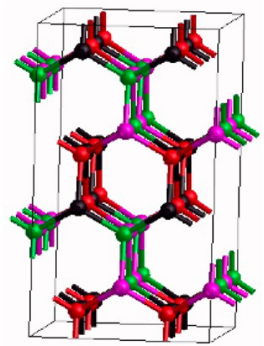

(c)

SiInCN
FIG. 6. (Color online) Predicted GS structures for: (a) $\mathrm{Si}_{3} \mathrm{GaC}_{3} \mathrm{~N}$ and $\mathrm{SiGa}_{3} \mathrm{CN}_{3}$, (b) $\mathrm{SiGaCN}$, and (c) $\mathrm{SiInCN}$ (online, black $=\mathrm{Si}$, green $=\mathrm{C}$; red $=\mathrm{N}$; gold $=\mathrm{Ga}$; violet $=\mathrm{In}$ online). Projections are close to the pseudohexagonal $c$-axis to emphasize differences in ordering within: (1) the (001) mixed-double-layers; and (2) ordering in cation-anion-cation. columns along $c_{H e x}$. In $\mathrm{Si}_{3} \mathrm{GaC}_{3} \mathrm{~N}$ and $\mathrm{SiGa}_{3} \mathrm{CN}_{3}$, mixed $(001)_{w}$-layers alternate with $\mathrm{SiC}$ and GaN-double-layers, respectively.

\section{B. Phase diagram calculations}

\section{SiC-AIN}

Phase diagram calculations for all three systems were performed with grand canonical monte carlo (MC) simulations using the multicomponent memc 2 code which is part of the ATAT package. ${ }^{21-23}$ Input parameters for memc2 were: a $14 \times 14 \times 8$ unit cell simulation box (1568 atoms); 1500 equilibration passes; 1500 Monte Carlo passes.

Two calculated SiC-AlN phase diagrams are shown in Fig. 8, and compared with the experiment-based miscibility gap of Zangvil and Ruh. ${ }^{10}$ The lower calculated curve (shown in blue online) was calculated with the weighted CE-fit described above. The upper dot-dashed-curve (shown in red online) was calculated with a CE-fit that only included $\{\Delta E<0.10 \mathrm{eV}\}$. Qualitatively, calculations and experiment agree in that both yield miscibility gaps, but there is great quantitative disparity: data in Zangvil and Ruh $^{10}$ suggest a consolute temperature of $\mathrm{T}_{C} \approx 2300 \mathrm{~K}$; versus $\mathrm{T}_{C} \approx 1200 \mathrm{~K}$ for the weighted CE-fit; and $\mathrm{T}_{C} \approx 1930 \mathrm{~K}$ for the $\{\Delta E<0.10 \mathrm{eV}\}$-fit.

\section{SiC-GaN}

The calculated phase diagram for the system SiC-GaN is shown in Fig. 9. Symmetry constraints on space groupsubgroup relationships were investigated with the ISOTROPY program, ${ }^{15,27}$ and consistent with the first-principles phase diagram calculations, all three ordered phases transform to the disordered wurtzite structure phase via first-order transitions. Transitions between the ordered phases are also first-order with broad two-phase fields.

\section{3. $\operatorname{SiC}-\operatorname{InN}$}

The calculated phase diagram for the system $\mathrm{SiC}-\mathrm{InN}$ is shown in Fig. 10. As for SiC-GaN, the ISOTROPY program ${ }^{27}$ and FPPD calculations predict a first-order transition between the wurtzite-structure phase and the ordered SiInCN, Cmc $2_{1}$, phase (Table I). Note that the order-disorder transition temper-
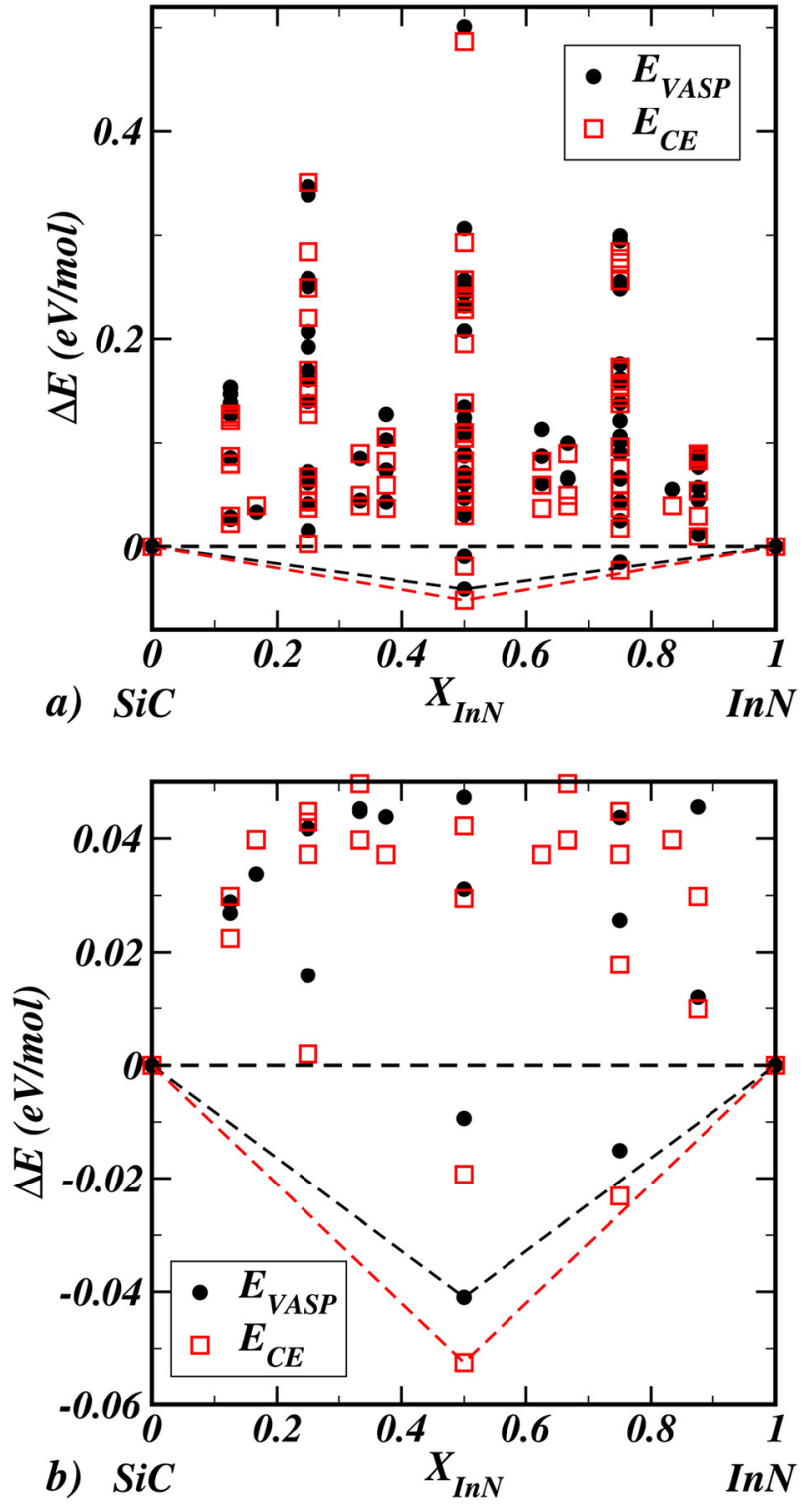

FIG. 7. (Color online) VASP and corresponding CE values for $\Delta E_{f}$ in SiCInN (color online: $\Delta E_{V A S P}=$ solid black circles; $\Delta E_{C E}=$ open red squares; dashed lines indicate convex hulls): (a) the energy distribution in SiC-GaN is similar to that in and $\mathrm{SiC}-\mathrm{GaN}$, but more compact with only three $\Delta E_{f}<0$.

ature, $\mathrm{T}_{O-D} \approx 1600 \mathrm{~K}$ at $X \approx 1 / 2$ is about a factor of two greater than the analogous temperature at $X \approx 1 / 2$ in $\mathrm{SiC}$ $\mathrm{GaN}, \mathrm{T}_{O-D} \approx 850 \mathrm{~K}$. Also, the calculated $\mathrm{T}_{C} \approx 1710 \mathrm{~K}$ at $X \approx 0.11$ in $\mathrm{SiC}$-InN, is more than a factor of two greater than the $\mathrm{T}_{O-D} \approx 850 \mathrm{~K}$ at $X \approx 1 / 2$ in SiC-GaN.

Topologically, the $\mathrm{SiC}$-InN diagram is quite similar to the $\mathrm{MgCO}_{3}-\mathrm{CaCO}_{3}$ and $\mathrm{MgCO}_{3}-\mathrm{CdCO}_{3}$ phase diagrams ${ }^{28}$ with the exception that, in the carbonate systems, the dolomite-structure phases at $X \approx 1 / 2$ transform to the disordered phase via second-order transitions.

\section{DISCUSSION}

\section{A. SiC-AIN}

The very low energies of $(\mathrm{SiC})_{m}(\mathrm{AlN})_{n} \ldots$ ordered layer structures (Figs. 1 and 2$)$ in $(\mathrm{SiC})_{1-X}(\mathrm{AlN})_{X}$ solid solutions, and the much higher energies of other ordered 


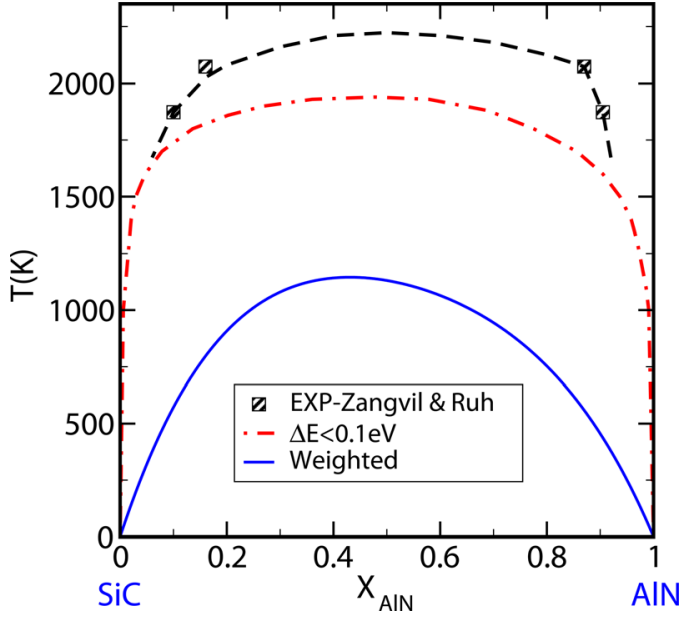

FIG. 8. (Color online) Comparison of experimental data from Zangvil and Ruh $^{10}$ with the results of first principles based calculations with a weightedfit cluster expansion (shown in blue online).

configurations, suggest that short-range order related to the layer-structures stabilize metastable solid solutions and therefore, enable synthesis of films and single crystals of arbitrary bulk composition. Similarly, the very high energies associated with various disordered states, helps to explain the very sluggish diffusion kinetics that are observed in this system; ${ }^{11}$ i.e., very high energies for states that arise in the disordered system imply high activation barriers for diffusion.

Ordered $(\mathrm{SiC})_{m}(\mathrm{AlN})_{n} \ldots$ layer-structures have low energies because they maximize the most stable, [Si]-(C) and [Al]-(N) bonds, within layers, while simultaneously allowing interlayer [Si]-(N) and [Al]-(C) bonds to relax toward ideal lengths. Such relaxations, parallel to $\mathrm{c}_{H E X}$, do not change $\mathrm{P}_{3} \mathrm{mc}$ symmetry. Also, note that such layer-structures may be regarded either as ordered superlattice structures (small values of $m, n, \ldots)$ or as a nano-micro phase separated state (large values of $m, n, \ldots$ ).

The occurrence of low-energy states with characteristic crystallography, $(\mathrm{SiC})_{1-X}(\mathrm{AlN})_{X}$ layer structures, raises interesting questions about the automation of FPPD calcula-

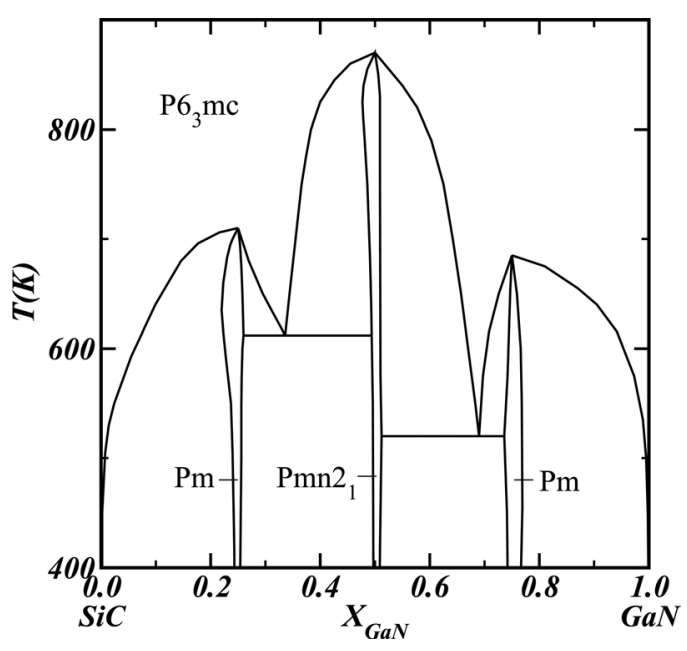

FIG. 9. Calculated phase diagram for the system SiC-GaN.

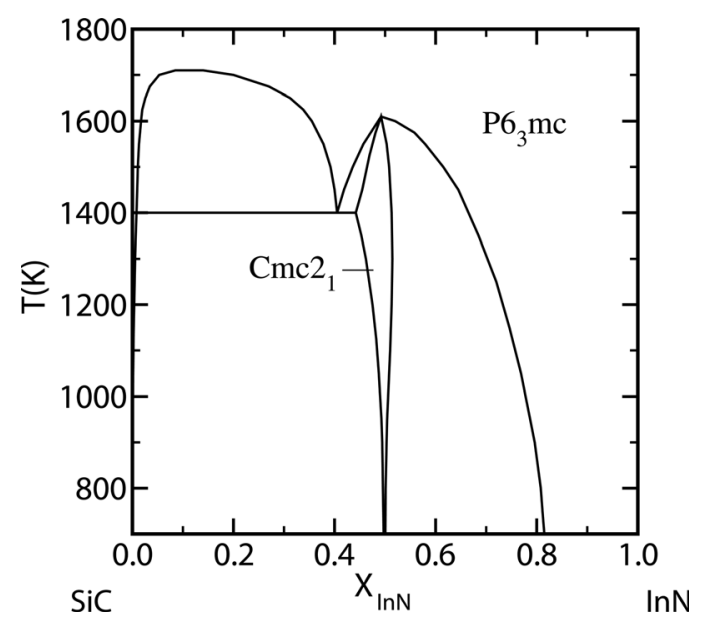

FIG. 10. Calculated phase diagram for the system SiC-InN.

tions: (1) how to automate the identification of such favored states and give extra weight to a ground-state search based on essential crystallographic features? (2) is unbiased structure-choice the best approach? (3) does it lead to the equilibrium diagram? (4) does the inclusion of many favored structures bias toward a phase diagram with underestimated transition temperatures (e.g., $\left.\mathrm{T}_{C}\right)$ ?

Typically, FPPD calculations overestimate the consolute temperatures $\left(\mathrm{T}_{C}\right)$ of miscibility gaps, and order-disorder transition temperatures $\left(\mathrm{T}_{O-D}\right)$ especially when, as here, the excess vibrational contribution to the free energy is ignored. In the SiC-AlN case however, that generalization may not hold. Including the vibrational contribution usually leads to a $5-15 \%$ reduction in $\mathrm{T}_{C}$ (Ref. 29), and similar results were obtained for the wurtzite-structure systems: AlN-GaN, GaNInN and AlN-InN (Ref. 30) (see however Ref. 31, for a case in which $\mathrm{T}_{C}$-reduction is closer to a $50 \%$ effect). Thus, assuming $10 \%$ vibrational free energy corrections for the calculated miscibility gaps in Fig. 8, one expects $\mathrm{T}_{C} \approx 1740 \mathrm{~K}$ for the $\{\Delta E<0.10 \mathrm{eV}\}$-fit, and $\mathrm{T}_{C} \approx 1100 \mathrm{~K}$ for the weighted CE-fit. Probably, the Zangvil and Ruh ${ }^{10}$ estimate of $\mathrm{T}_{C} \approx 2300 \mathrm{~K}$ is too high, but uncertainties in the calculations presented make this a weak prediction.

\section{B. SiC-GaN and SiC-InN}

Comparing Figs. 5 and 7 clarifies the large difference between the three $\mathrm{T}_{O-D}$ in SiC-GaN, versus $\mathrm{T}_{O-D}$ and $\mathrm{T}_{C}$ in $\mathrm{SiC}-\mathrm{InN}$. In SiC-GaN, there are $24 \Delta E_{V A S P}<0$, and in SiCInN, there are only three, and only five with $\Delta E_{V A S P}<0.02$ $\mathrm{eV}$. Thus, chemical disorder is energetically cheaper in SiC$\mathrm{GaN}$, and ordering-transition temperatures are lower by about a factor of two.

\section{CONCLUSIONS}

Experimental observations were interpreted as indicating a miscibility gap in the wurtzite-structure $\mathrm{SiC}$-AlN solid solution with a consolute temperature of $\mathrm{T}_{C} \approx 2300 \mathrm{~K}$ (Refs. 10 and 11). The weighted CE-fit and $\{\Delta E<0.10 \mathrm{eV}\}$-fit calculations presented here predict lower consolute temperatures, but with great uncertainty. 
Total energy calculations predict the presence of lowenergy metastable layer-structures of the form $(\mathrm{SiC})_{m}(\mathrm{AlN})_{n}$ $(\mathrm{SiC})_{o}(\mathrm{AlN})_{p} \ldots$, in which $m-, o^{-}, \ldots$ layers of $\mathrm{SiC}, \perp$ to $c_{\mathrm{Hex}}$ alternate with $n-, p-, \ldots$ layers of $\mathrm{AlN}, \perp$ to $c_{\mathrm{Hex}}$. Formation energies for layer structures in which $(\mathrm{SiN})$ and $(\mathrm{AlC}) \mathrm{nn}-$ layers are maximized (i.e., $\mathrm{Si}-\mathrm{N}$ and $\mathrm{Al}-\mathrm{C}$ bonds are maximized) have much higher energies, $50 \mathrm{~kJ} / \mathrm{mol}$ to $75 \mathrm{~kJ} / \mathrm{mol}$ higher, than the $(\mathrm{SiC})_{m}(\mathrm{AlN})_{n}$ layer-structures. It seems likely that the relative ease with which one can synthesize apparently homogeneous films and single crystals of $(\mathrm{SiC})_{1-X}(\mathrm{AlN})_{X}$ at arbitrary $X$, reflects a reduction in total energy that derives from short-range order based on $(\mathrm{SiC})_{m}$ $(\mathrm{AlN})_{n}(\mathrm{SiC})_{o}(\mathrm{AlN})_{p} \ldots$ layer structures. This characteristic local structure helps to explain the very sluggish kinetics in this system: diffusion barriers through highly stable SiC- or AlN-double layers are very high.

Three stable ordered phases are predicted in SiC-GaN, and one in SiC-InN. Disordering of the SiC-GaN ordered phases occurs at temperatures that are about a factor of two lower than the disordering temperatures, $\mathrm{T}_{O-D}$ and $\mathrm{T}_{C}$ in $\mathrm{SiC}-\mathrm{InN}$.

Cluster expansions of hybrid-functional-based band gaps indicate only a small short-range-order in (clustering) effect on band gaps in SiC-AIN solid solutions, but very significant effects in SiC-GaN, and to a lesser extent in SiC-InN.

The topology of the SiC-InN phase diagram is similar to those for carbonate systems such as $\mathrm{MgCO}_{3}-\mathrm{CaCO}_{3}$ and $\mathrm{MgCO}_{3}-\mathrm{CdCO}_{3}$ (Ref. 28), with the exception that in $\mathrm{SiC}-\mathrm{InN}$, the order-disorder transition at $X \approx 1 / 2$ and $\mathrm{T} \approx 1600 \mathrm{~K}$, is a first-order transition rather than a second-order transition as in the carbonates.

\section{ACKNOWLEDGMENTS}

This work is supported by NIST, the Department of Energy National Nuclear Security Administration under Award No. DE-FC52-08NA28613, the National Science Foundation under Grant No. DMR-0907669, and through TeraGrid resources at TACC under Grant No. TG-DMR050013N.

${ }^{1}$ Z. Gu, L. Du, J. H. Edgar, E. A. Payzant, L. Walker, R. Liu and M. H. Engelhard, MRS Internet J. Nitride Semicond. Res. 10(5), 1 (2005).

${ }^{2}$ J. H. Edgar, Z. Gu, L. Gu, and D. Smith, Phys. Status Solidi A 203(15), 3720 (2006).
${ }^{3}$ R. Roucka, J. Tolle, A. V. G. Chizmeshya, P. A. Crozier, D. J. Smith, I. S. T. Tsong, and J. Kouvetakis, Phys. Rev. Lett. 88, 206102 (2002).

${ }^{4}$ J. Tolle, R. Roucka, A. V. G. Chizmeshya, P. A. Crozier, D. J. Smith, I. S. T. Tsong, and J. Kouvetakis, Solid State Sci. 41509 (2002).

${ }^{5}$ The term quasibinary is used rather than pseudobinary because the calculated phasediagrams obey a binary phase rule, although the real system may deviate slightly from this ideal.

${ }^{6}$ P. B. Perry and R. F. Rutz, Appl. Phys. Lett. 33, 319 (1978).

${ }^{7}$ B. Monemar, Phys. Rev. B 10, 676 (1974).

${ }^{8}$ T. L. Tanesley and C. P. Foley, J. Appl. Phys. 59, 3241 (1986).

${ }^{9}$ N. B. Singh, B. Wagner, A. Berghmans, D. J. Knuteson, S. McLaughlin, D. Kahler, D. Thomson, and M. King, Cryst. Growth Des. 10, 3508 (2010).

${ }^{10}$ A. Zangvil and R. Ruh, J. Am. Ceram. Soc. 71(10), 884 (1988).

${ }^{11}$ W. Rafaniello, M. R. Plichta, and A. V. Virkar, J. Am. Ceram. Soc. 66(4), 272 (1983); B. Paulus, F-J Shi, and H. Stoll, J. Phys. Condens. Mater. 9, 2745 (1997).

${ }^{12}$ Z. Q. Liu and J. Ni, Eur. Phys. J. B 59, 29 (2007).

${ }^{13}$ G. Kresse, and J. Hafner, Phys. Rev. B 47, 558 (1993); G. Kresse, Ph. D thesis, Technische Universität Wien, 1993; G. Kresse, Phys. Rev. B 49(14) 251 (1994); G. Kresse and J. Furthmüller, Comput. Mater. Sci. 6, 15 (1996); G. Kresse and J. Furthmüller, Phys. Rev. B 54, 11169 (1996); See http://tph.tuwien.ac.at/vasp/guide/vasp.html. The Al, $\mathrm{N}_{h}$, Si and $\mathrm{C}_{s}$ PAW potentials were used in this work.

${ }^{14}$ D. Vanderbilt, Phys. Rev. B 41, 7892 (1990).

${ }^{15}$ Reference to specific software packages does not imply a NIST endorsement.

${ }^{16}$ J. M. Sanchez, F. Ducastelle, and D. Gratias, Physica A 128, 334 (1984).

${ }^{17}$ B. P. Burton, Am. Mineral. 70, 1027 (1985).

${ }^{18}$ G. Ceder, G. D. Garbulsky, D. Avis, and A. Fukuda, Phys. Rev. B 49, 1 (1994).

${ }^{19}$ D. de Fontaine, Solid State Physics 4733 (1994).

${ }^{20}$ R. McCormack and D. deFontaine, Phys. Rev B, 54, 9746 (1996).

${ }^{21}$ A. van de Walle, M. Asta, and G. Ceder, CALPHAD: Comput. Coupling Phase DiagramsThermochem. 26539 (2002).

${ }^{22}$ A. van de Walle and G. Ceder, J. Phase Equilib. 23348 (2002).

${ }^{23}$ A. van de Walle and M. Asta, Modell. Simul. Mater. Sci. Eng. 10521 (2002).

${ }^{24}$ J. Heyd, G. E. Scuseria, and M. Ernzerhof, J. Chem. Phys. 118, 8207 (2003); J. Heyd, G. E. Scuseria, and M. Ernzerhof, ibid. 124, 219906 (2006).

${ }^{25}$ A. V. Krukau, O. A. Vydrov, A. F. Izmaylov, and G. E. Scuseria, J. Chem. Phys. 125, 224106 (2006); H. T. Stokes and D. M. Hatch, J. Appl. Crystallogr. 38, 237 (2005); See http://stokes.byu.edu/cgi-bin/iso/findsym.cgi.

${ }^{26} \mathrm{P}$. Villars and L. D. Calvert, Pearson's Handbook of Crystallographic Data for Intermetallic Phases (American Society for Metals, Metals Park, OH 1985), Vol. 1.

${ }^{27}$ H. T. Stokes, D. M. Hatch, and B. J. Campbell, (2007). ISOTROPY, see http://stokes.byu.edu/isotropy.html.

${ }^{28}$ B. P. Burton and A. Van de Walle, Phys. Chem. Miner. 30, 88 (2003).

${ }^{29}$ A. van de Walle and G. Ceder, Rev. Mod. Phys. 74, 11 (2002).

${ }^{30}$ B. P. Burton, A. van de Walle, and U. Kattner, J. Appl. Phys. 100, 113528 (2006).

${ }^{31}$ B. P. Burton and A. van de Walle, Chem. Geol. 225, 222 (2006). 